\title{
Hadronic Cross Section Measurements at Belle and perspec- tives for BELLE-II
}

\author{
Shwartz B. ${ }^{1,2, \star}$ for Belle collaboration \\ ${ }^{1}$ Budker Institute of Nuclear Physics \\ ${ }^{2}$ Novosibirsk State University, Novosibisrsk, Russia
}

\begin{abstract}
Large data amount, about $1 \mathrm{ab}^{-1}$ of integrated luminosity, collected in experiments with the Belle detector provided a good possibilities for precise measurements of the hadronic cross sections in $e^{+} e^{-}$annihilation. Main results obtained in this field with the Belle detector as well as perspectives of the new experiments with the Belle II detector at the SuperKEKB collider is discussed in this report.
\end{abstract}

\section{Introduction}

Experiments at the KEKB $e^{+} e^{-}$energy-asymmetric collider [1] with world highest luminosity, $2.1 \times$ $10^{34} \mathrm{~cm}^{-2} \mathrm{~s}^{-1}$, continued with the Belle detector [2] from 1999 to 2010 . The total integrated luminosity collected in these experiments in the centre-of-mass energy range within $\Upsilon$-meson family exceeded $1000 \mathrm{fb}^{-1}$. The main target of the Belle experiment was to discover the CP-violation (CPV) in B meson decays and to measure its parameters. This goal was achieved in 2001 when the time dependent CP asymmetry was observed in the decay $B^{0} \rightarrow J / \Psi K^{0}$ decay [3]. However, in addition to the main task, many other important results listed below were obtained, including precise measurement of the hadronic cross sections in $\gamma \gamma$ and $e^{+} e^{-}$processes. This wide researches area became possible due to the clean and well defined initial state in the $e^{+} e^{-}$experiments as well as large KEKB luminosity and high quality of the Belle detector.

In this note we discuss results of the study of the hadrons production in $e^{+} e^{-}$annihilation obtained by the Belle experiment via direct energy scan and by the ISR approach. Precise measurements of the total cross sections of the $e^{+} e^{-}$annihilation into hadrons and detail study the final states produce important information about quark interactions, spectroscopy of their bound states and provide a basis for the calculations of the hadronic contributions to the fundamental parameters, like muon $(g-2)$ value or $\alpha\left(M_{Z}^{2}\right)$.

\section{Measurements of $R_{b}$ via energy scan}

The total cross sections of $e^{+} e^{-} \rightarrow b \bar{b}$ and $e^{+} e^{-} \rightarrow \Upsilon_{n S} \pi^{+} \pi^{-}(n=1,2,3)$ processes were measured by the Belle collaboration in the energy range from 10.63 to $11.05 \mathrm{GeV}$ using the direct high luminosity energy scan [4]. Results of these work expressed as the ratio, $R$, of the measured cross section to the $\mu^{+} \mu^{-}$Born cross section, $\sigma_{\mu \mu}^{0}=\left(4 \pi \alpha^{2}\right) / 3 s=86.85[\mathrm{nb}] / s\left[\mathrm{GeV}^{2}\right]$, are presented in the Fig. 1 .

\footnotetext{
^e-mail: shwartz@inp.nsk.su
} 


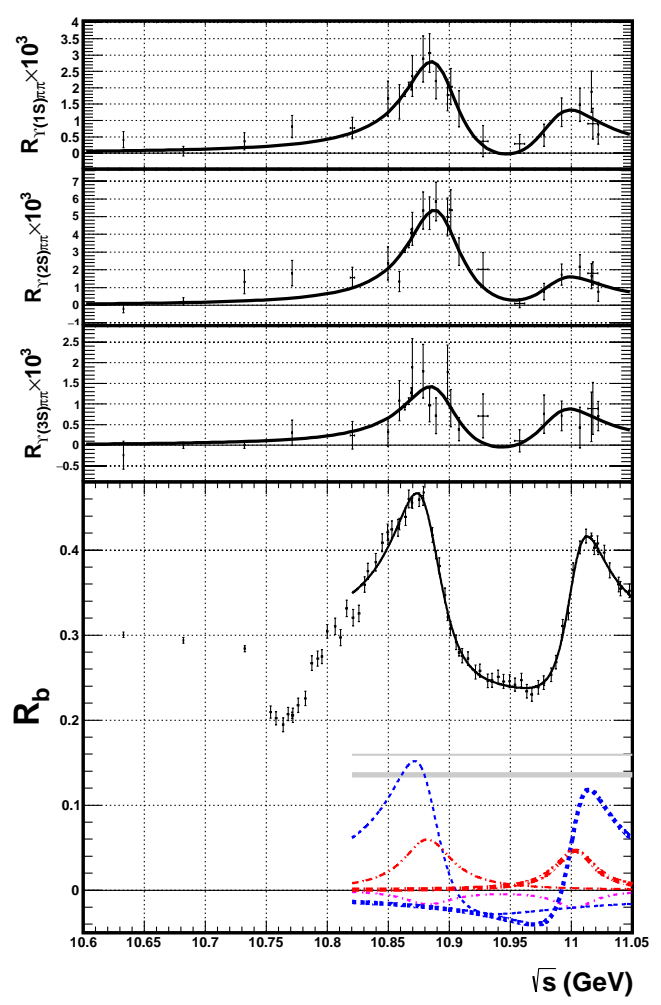

Figure 1. Upper three plots show $R_{\Upsilon_{n S} \pi^{+} \pi^{-}}$data measured in this work with corresponding fitting curves. Bottom plot: $R_{b}$ results obtained in this work with the fit curve (solid) and its components. Horizontal lines correspond to $\left|A_{i c}\right|^{2}$ (thin) and $\left|A_{c}\right|^{2}$ (thick) constants. Other curves present: $|f|^{2}$ (dot-dot-dash), cross terms of resonances with $A_{c}$ (dashed) and two-resonance cross term (dot-dash). Thin and thick curves correspond to $\Upsilon_{5 S}$ and $\Upsilon_{6 S}$ respectively. Error bars include the statistical and uncorrelated systematic uncertainties [4].

Since no non-resonant contributions were found in the $\Upsilon(n S) \pi^{+} \pi^{-}$cross section these were fitted by the squared module of the sum of $\Upsilon_{5 S}(\Upsilon(10860))$ and $\Upsilon_{6 S}(\Upsilon(11020))$ meson amplitudes that provided precise values of the parameters of these resonances: $M_{5 S}=10891.1 \pm 3.2_{-1.7}^{+0.6} \mathrm{MeV} / \mathrm{c}^{2}$, $\Gamma_{5 S}=53.7_{-5.6-5.4}^{+7.1+1.3} \mathrm{MeV}, M_{6 S}=10987.5_{-2.5-2.1}^{+6.4+9.0} \mathrm{MeV} / \mathrm{c}^{2}, \Gamma_{5 S}=61_{-19-20}^{+9+2} \mathrm{MeV}$, phase difference $\Delta \varphi(6 S-5 S)=-1.0 \pm 0.4_{-0.1}^{+1.4}$. The first uncertainty is statistical, the second one is systematic.

Values of $R_{b}=\sigma\left(e^{+} e^{-} \stackrel{-0}{\rightarrow} b \bar{b}\right) / \sigma_{\mu \mu}^{0}$ obtained in this experiment were fitted with the formula:

$$
R(S)=\left|A_{i c}\right|^{2}+\left|A_{c}+A_{5 S} e^{i \varphi_{5 S}} f_{5 S}(s)+A_{6 S} e^{i \varphi_{6 S}} f_{6 S}(s)\right|^{2},
$$

where $\left.f_{n S}=M_{n S} \Gamma_{(n S}\right) /\left(s-M_{n S}^{2}+i M_{n S} \Gamma_{n S}\right)$ while $A_{c}$ and $A_{i c}$ are coherent and incoherent constant amplitudes, respectively. Obtained optimal curves are shown the in Fig. 1 as well. Parameters obtained by this fit are consistent with the quoted above.

\section{Measurements of the charm production cross section via ISR}

In the last years a lot of new data were obtained using initial state radiation (ISR) processes [5] at $B-$, $c-\tau$ and $\phi$-factories, by the BaBar, Belle, KLOE and BES III experiments. After emission of hard photon $e^{+} e^{-}$pair can acquire any invariant mass below the energy of the experiment. That means that one can study the processes of $e^{+} e^{-}$annihilation in the entire range from the threshold to the experiment energy. The ISR photon is mostly emitted at a small angle when it cannot be detected by the detector. In case of processes with high invariant mass of the final state, a study can be performed without photon detection. A lot of results were obtained by this way by the Belle collaboration in the 
charm sector [6-18]. However, interesting results were obtained also for the processes $e^{+} e^{-} \rightarrow \phi \pi^{+} \pi^{-}$ and $e^{+} e^{-} \rightarrow \phi f_{0}(980)$ in the energy range from 1.5 to $3 \mathrm{GeV}$ [19] .

The $R_{c}$ experimental values obtained by summing of the cross sections measured by the Belle experiment [20] in comparison with BES III results [21] are presented in the Fig. 2. Contributions of $D^{+} D^{*-}, D^{*+} D^{*-}, D^{0} D^{-} \pi^{+}$and $D^{0} D^{*-} \pi^{+}$are scaled following isospin symmetry. BES III data were evaluated as the difference $R_{c}=R_{t o t}-R_{u d s}$. Belle results on charmonium-like XYZ states is presented

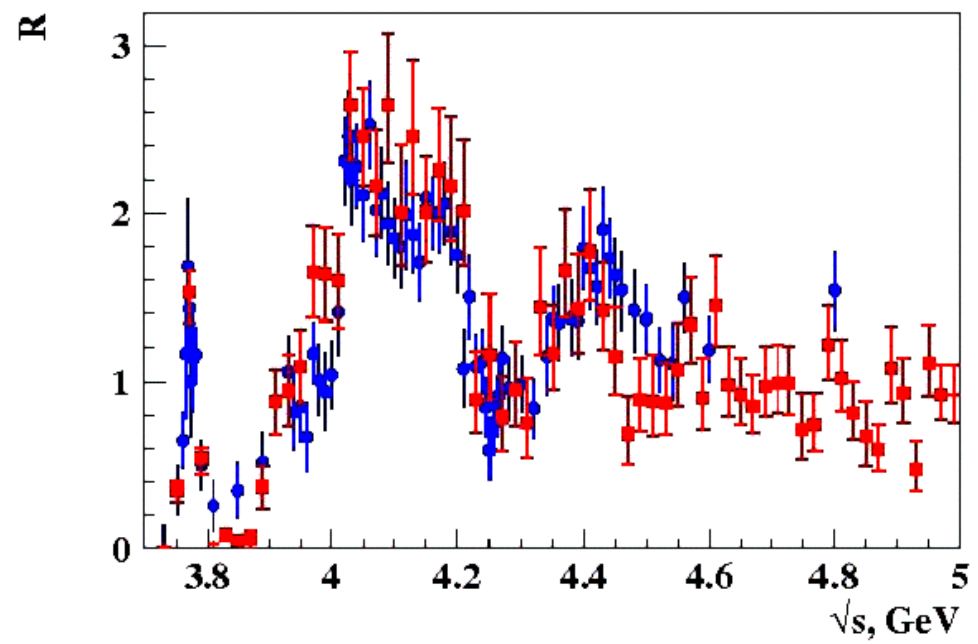

Figure 2. $R_{c}$ experimental values obtained by summing of the cross sections measured by the Belle experiment [20] (squares) in comparison with BES III results [21] (circles).

by R.Mizuk [22] at this conference.

\section{Study of hadronic cross sections at low energies}

Precise measurements of cross sections of $e^{+} e^{-}$annihilation into hadrons at low energies are highly demanded. These measurements as well as a detail study of the final states provide important information about interactions of light quarks and spectroscopy of their bound states. Precise determination of the total hadron production cross section, characterized by the ratio $R_{h}=\sigma\left(e^{+} e^{-} \rightarrow\right.$ hadrons $) / \sigma_{\mu \mu}^{0}$, is necessary for calculations of the muon anomalous magnetic moment, $a_{\mu}=\left(g_{\mu}-2\right) / 2$, as well as running electromagnetic and strong interaction constants. A special interest to the $a_{\mu}$ was induced by the difference at the level of 3.5 standard deviation between experimental $a_{\mu}$ value [23] and theoretical calculations that includes the contribution from the hadronic vacuum polarization. Recent review of the status of theoretical calculation is given in [24].

At present the accuracy of the mentioned difference, $\Delta a_{\mu}=a_{\mu}(\exp )-a_{\mu}(t h)$, is mainly determined by the experimental precision, $a_{\mu}=(11659208.9 \pm 6.3) \cdot 10^{-10}$ [23]. However, recently new experiment (FNAL-E989) aiming for the improvement of the precision by factor three started in FNAL [25]. This experiment is based on the same principles as the previous one and reuses the same muon storage ring. Another experiment based on a different approach having the same target precision is in preparation at J-PARC [26]. Then, since the accuracy of the theoretical calculations of $a_{\mu}$ is dominated by the precision of the hadronic contribution, $a_{\mu}^{H}$ the uncertainty of this value should be reduced by the same factor. 
The BaBar experiment achieved the level of systematic accuracy in the $\pi^{+} \pi^{-}$cross section of about $1 \%$ [27] at the measurement via ISR. Two other experiments using the ISR approach, KLOE and BES III, recently published results on the $\pi^{+} \pi^{-}$channel with the same accuracy citekloe-pipi,bes-pipi. However, there are certain discrepancies between data of different experiments exceeding the stated uncertainties. Thus, new and more precise measurements of the hadronic cross section are needed.

For low $M_{i n v}^{h}$ the hard photon energy as well as the total energy of the hadronic system is equal to about half of the energy of experiment. Then the pions move in the narrow cone at opposite direction to the photon with energies in the $\mathrm{GeV}$ range. These kinematical features are very different from multiparticle events typical for B-factories and require special study of the detection efficiency as well as track reconstruction efficiency. A proper trigger arrangement is extremely important for ISR events study. However, in the Belle case the trigger system was not sophisticated enough and tagged low mass ISR events as Bhabha ones with rather large probability. Since Bhabha events were prescaled this results in a low trigger efficiency and its considerable instability. That was a main problem which limited precise low mass hadronic cross sections measurements via ISR at the Belle.

In the Belle II experiment the Bhabha tagging at the level 1 trigger will be drastically improved using accurate trigger cell clusters determination in the trigger electronics. In the L1 trigger we will have several independent trigger modes, charged and neutral, to provide careful measurement and monitoring of the trigger efficiency from the experimental data during experiment.

\section{Super KEKB and Belle II}

New energy-asymmetric $e^{+} e^{-}$collider, SuperKEKB, is under commissioning now at KEK (Japan) [30]. The luminosity of this collider will exceed the previously achieved by KEKB [1] by about 40 times, amounting to $8 \times 10^{35} \mathrm{~cm}^{-2} \mathrm{~s}^{-1}$. New experiments with the Belle II detector in the center-ofmass (CMS) energy range within $\Upsilon$-meson family $(9-11.5 \mathrm{GeV})$ will continue and widen the studies began at the previous experiments. The detail description of the Belle II project can be found in [30]. Schematic view of the Belle II detector is presented in Fig. 3.

The tracking system of the Belle II detector consists of the Vertex Detector (VXD) and the Central Drift Chamber (CDC). The VXD contains the two layer Pixel Detector (PXD) and four layer Silicon Vertex Detector (SVD). The PXD is based on DEPFET technology which allows to produce very thin (down to $50 \mu \mathrm{m}$ ) sensors.

The central drift chamber (CDC) provides track reconstruction and its precise momentum measurement; a measurement of the ionization losses of the charged particles for the identification purposes; a generation of the signals for the trigger system. The momentum resolution of the Belle II with CDC and SVD is $\sigma_{p_{t}} / p_{t}=0.11 \% \cdot p_{t}[\mathrm{GeV} / \mathrm{c}] \oplus 0.30 \% / \beta$, which it much better than that for the Belle detector. The expected $d E / d x$ resolution is about $5 \%$.

Main charged particle identification (PID) sub-systems are based on the Cherenkov ring detection. In the barrel part of the Belle II detector the time-of-propagation (TOP) counters [31] are employed. This system will provide a good pion-kaon separation in the momentum range up to $3.5 \mathrm{GeV} / \mathrm{c}$. Identification of charged particles in the forward endcap region will be performed by the proximityfocusing aerogel ring-imaging Cherenkov detector (ARICH). This system allows to obtain $99 \%$ of kaon identification efficiency at $1 \%$ of pion misidentification for particles with $4 \mathrm{GeV} / \mathrm{c}$ momentum.

The existent CsI(Tl) crystal Electromagnetic Calorimeter (ECL), are planned to be used together with the same PIN photo-diodes, preamplifiers and cables connected them to shapers. The baseline of the upgrade of the ECL is the electronics modification following the general strategy of the Belle upgrade.

A superconducting solenoid providing a magnetic field of $1.5 \mathrm{~T}$ as well as an iron yoke will be reused from the Belle detector. The Belle KL\&Muon detector (KLM) is integrated with the iron yoke 


\section{Belle II Detector}

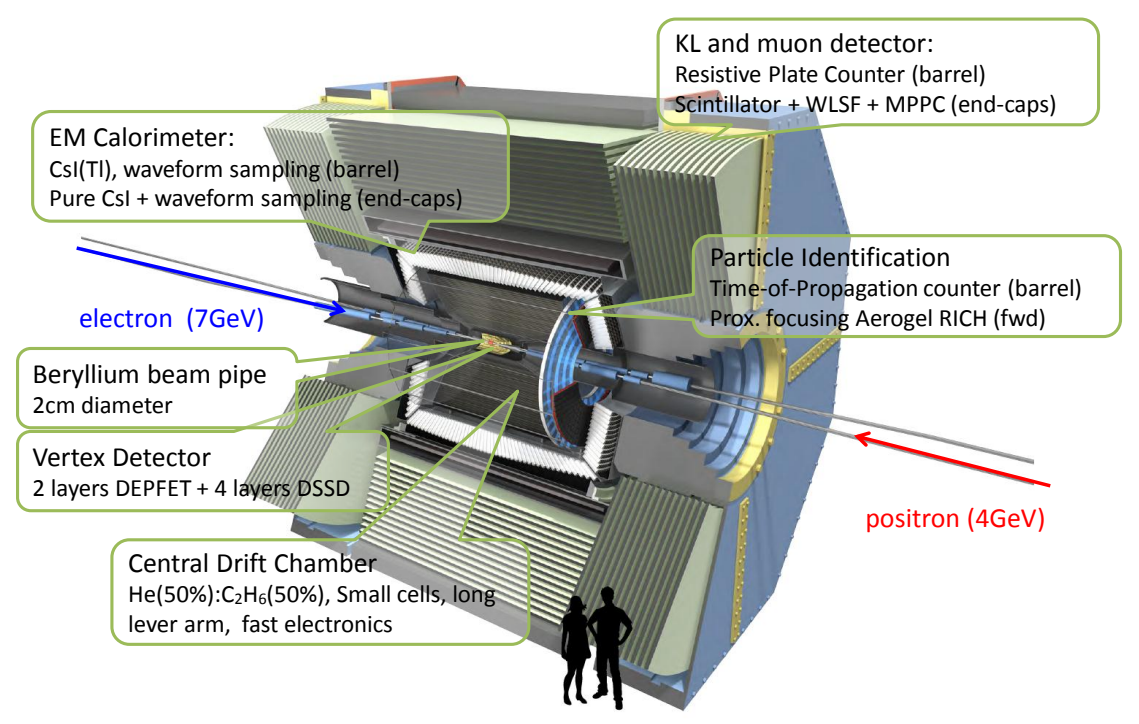

Figure 3. Schematic view of the Belle II detector.

of the magnet. For the Belle II detector the end cap KLM parts as well as two innermost layers of the barrel KLM part are replaced to the system based on the plastic scintillators. Remaining barrel part keeps the RPC system.

Experiments with the Belle II detector will start in 2018. It is expected to collect $10 \mathrm{fb}^{-1}$ of integrated luminosity in 2021 and $50 \mathrm{fb}^{-1}$ in 2024. The project parameters of the tracking and particle identification at the Belle II are much better than those were at the Belle and BaBar. Thus, we can hope for the drastic improvement of the corresponding contributions to the systematic uncertainties. High data statistics expected at Belle II experiment will provide a possibility of the careful comparison of the simulation and experimental efficiencies and determination of the corresponding corrections which should improve systematics as well.

\section{Conclusions}

Last decade demonstrated the fruitfulness of the flavor "factories" for hadronic cross section measurements via ISR as well as by the direct scan.

At present SuperKEKB/Belle II project is in commissioning. Very high expected luminosity of this experiment provides a possibility of the precise measurements of the hadronic cross section in a wide energy range from production thershold to $11.5 \mathrm{GeV}$.

We hope that high statistics and improved detector will help to reduce considerably systematic uncertainties.

To provide accurate data, especially for low mass range, we need to care about the proper trigger system and to prepare instruments to control stability of the charge particles and photon reconstruction efficiency during experiment. 
This work is supported by the Russian Science Foundation (project N 14-50-00080).

\section{References}

[1] S. Kurokawa et al., Nucl. Instr. and Meth. A, 499, 1 (2003).

[2] A. Abashian et al., Nucl. Instr. and Meth. A, 479, 117 (2002).

[3] Belle Collab. (A. Abashian et al.), Phys. Rev. Lett. 86, 2509 (2001);

[4] D. Santel et al., Phys. Rev. D 93, 011101(R) (2016).

[5] V.N. Baier, V.A.Khoze, Sov. Phys. JETP 21, 1145 (1965);

S. Binner, H.J. Kuhn, K. Melnikov, Phys. Lett. B 459, 279 (1999);

M.Benayoun et al., Mod. Phys. Lett. A, 142605 (1999).

[6] G. Pakhlova et al. (Belle Collaboration), Phys. Rev. Lett. 98, 092001 (2007).

[7] G. Pakhlova et al. (Belle Collaboration), Phys. Rev. Lett. 100, 062001 (2008).

[8] G. Pakhlova et al. (Belle Collaboration), Phys. Rev. Lett. 101, 172001 (2008).

[9] G. Pakhlova et al. (Belle Collaboration), Phys. Rev. D 80, 091101(R) (2009).

[10] G. Pakhlova et al. (Belle Collaboration), Phys. Rev. D 77, 011103(R) (2008).

[11] C.Z. Yuan et al. (Belle Collaboration), Phys. Rev. Lett.99, 182004 (2007).

[12] X.L. Wang et al. (Belle Collaboration), Phys. Rev. Lett. 99, 142002 (2007).

[13] C.Z. Yuan et al. (Belle Collaboration), Phys. Rev. D 77, 011105(R) 2008.

[14] X.L. Wang et al. (Belle Collaboration), Phys. Rev. D 87, 051101(R) 2013.

[15] Z.Q. Liu et al. (Belle Collaboration), Phys. Rev. Lett. 110, 252002 (2013); Erratum Phys. Rev. Lett. 111, 019901 (2013).

[16] C.P. Shen et al. (Belle Collaboration), Phys. Rev. D 89, 072015 (2014).

[17] X.L. Wang et al. (Belle Collaboration), Phys. Rev. D 91, 112007 (2015).

[18] Y.L. Han et al. (Belle Collaboration), Phys. Rev. D92, 012011 (2015).

[19] C.P. Shen et al. (Belle Collaboration) Phys. Rev. D 80, 031101(R) (2009).

[20] G.V. Pakhlova, P.N. Pakhlov, S.I. Eidelman, Phys.Usp. 53, 219 (2010); Usp.Fiz.Nauk 180, 225 (2010).

[21] M. Ablikim et al. (BES Collab.), Phys. Lett. B 660315 (2008).

[22] R. Mizuk, Recent results on XYZ physics from BELLE, International Workshop on $e^{+} e^{-}$collisions from Phi to Psi 2017.

[23] G.W. Bennett et al., Phys. Rev. D 73, 072003 (2006).

[24] F. Jegerlehner, DESY-17-058, HU-EP-17-12, arXiv:1705.00263v1 [hep-ph] (2017).

[25] D.W. Hertzog EPJ Web Conf. 118, 01015 (2016).

[26] N. Saito et al., AIP Conf.Proc. 1467, 45 (2012).

[27] J.P.Lees et al. (BaBar Collaboration), Phys. Rev. D 86, 032013 (2012).

[28] D. Babusci et al. (KLOE Collaboration), Phys. Lett. B 720, 336 (2013).

[29] M. Ablikim et al. (BESIII Collaboration), Phys. Lett. B 753, 629 (2016).

[30] T. Abe et al., Belle II technical design report, KEK Report 2010-1 (2010).

[31] K. Inami et al., Nucl. Instr. and Meth. A 595, 96 (2008). 